\title{
Rapid detection and differentiation of mycobacterial species using a multiplex PCR system
}

\author{
Andrea Santos Lima ${ }^{[1]}$, Rafael Silva Duarte ${ }^{[2]}, L_{i}$ lian Maria Lapa Montenegro ${ }^{[1]}$ \\ and Haiana Charifker Schindler ${ }^{[1]}$
}

[1]. Departamento de Imunologia, Centro de Pesquisas Aggeu Magalhães, Fundação Oswaldo Cruz, Recife, PE. [2]. Instituto de Microbiologia, Universidade Federal do Rio Janeiro, Rio de Janeiro, RJ.

\begin{abstract}
Introduction: The early diagnosis of mycobacterial infections is a critical step for initiating treatment and curing the patient. Molecular analytical methods have led to considerable improvements in the speed and accuracy of mycobacteria detection. Methods: The purpose of this study was to evaluate a multiplex polymerase chain reaction system using mycobacterial strains as an auxiliary tool in the differential diagnosis of tuberculosis and diseases caused by nontuberculous mycobacteria (NTM) Results: Forty mycobacterial strains isolated from pulmonary and extrapulmonary origin specimens from 37 patients diagnosed with tuberculosis were processed. Using phenotypic and biochemical characteristics of the 40 mycobacteria isolated in LJ medium, $57.5 \%(\mathrm{n}=23)$ were characterized as the Mycobacterium tuberculosis complex (MTBC) and $20 \%(\mathrm{n}=8)$ as nontuberculous mycobacteria (NTM), with $22.5 \%(\mathrm{n}=9)$ of the results being inconclusive. When the results of the phenotypic and biochemical tests in 30 strains of mycobacteria were compared with the results of the multiplex PCR, there was $100 \%$ concordance in the identification of the MTBC and NTM species, respectively. A total of $32.5 \%(n=13)$ of the samples in multiplex PCR exhibited a molecular pattern consistent with NTM, thus disagreeing with the final diagnosis from the attending physician. Conclusions: Multiplex PCR can be used as a differential method for determining TB infections caused by NTM a valuable tool in reducing the time necessary to make clinical diagnoses and begin treatment. It is also useful for identifying species that were previously not identifiable using conventional biochemical and phenotypic techniques.
\end{abstract}

Keywords: Mycobacterium tuberculosis complex. Nontuberculous mycobacteria. Diagnosis.

Polymerase chain reaction. Multiplex.

\section{INTRODUCTION}

The ability of nontuberculous mycobacteria (NTM) to cause disease is clearly described in the literature, and its importance is increasing progressively, with an increasing amount of various species being cultured and isolated in laboratories ${ }^{1-3}$.

In Brazil, the NTM species most frequently associated with lung disease are Mycobacterium kasasii and Mycobacterium avium. Other species, such as Mycobacterium xenopi, Mycobacterium malmoense, Mycobacterium lentiflavum, Mycobacterium abscessus and Mycobacterium szulgai, have occasionally been isolated in cultures ${ }^{4.5}$. In 2011 the National Agency for Sanitary Surveillance (ANVISA) published the number of cases of infections by rapidly growing mycobacteria (RGMs) obtained in the last eleven years in Brazil.

Data also suggest that this disease occurs in subjects undergoing invasive procedures, mostly surgical cases

\footnotetext{
Address to: $\mathrm{Dr}^{\mathrm{a}}$ Andrea Santos Lima. Dept ${ }^{\mathrm{o}}$ de Imunologia/CPqAM/FIOCRUZ Av. Moraes Rego s/n, Cidade Universitária, 50670-420 Recife, PE, Brasil. Phone: 5581 2101-2569; Fax: 5581 3453-1911

e-mail: andreasantoslima@hotmail.com

Received 6 May 2013

Accepted 22 July 2013
}

guided by video, where the instruments are subjected to high levels of sterilization in glutaraldehyde solution. From 1998 to 2009 , there were 2,520 reported cases of postsurgical infections related to rapidly growing mycobacteria, including Mycobacterium fortuitum, Mycobacterium chelonae, M. abscessus and Mycobacterium massiliense, which were distributed predominantly in private hospitals throughout the country. Cases have been reported in 23 states of Brazil, with $97.8 \%$ of the cases concentrated within 10 states $^{6}$. The diseases caused by NTMs are not compulsorily notifiable, except where the infection occurred during surgical procedures; however, several studies in Brazil have noted isolated strains of nontuberculous mycobacteria in pulmonary and extrapulmonary clinical samples from patients with suspected tuberculosis ${ }^{4,7,8}$.

The differential diagnosis between tuberculosis (TB) and diseases caused by NTMs is of great importance because the epidemiology, treatment and prognosis are different. Conventional diagnostics present numerous difficulties as clinical symptoms are generally similar and non-specific smear testing has limited sensitivity and cannot differentiate the species of mycobacteria.

For many years, the identification of mycobacteria was undertaken by gathering phenotypic results and biochemical test results of isolated species in culture medium ${ }^{9,10}$. In the last 20 or 30 years, with the increasing need to identify a greater number of species and also for early diagnosis, new methods 
have been developed to enable rapid and accurate identification of mycobacterial species ${ }^{10-12}$. In Brazil, the PRA-hsp65 technique proposed by Telenti et al. ${ }^{13}$, and Devallois et al. ${ }^{14}$ has enabled the identification of several species of NTM, the results of which correlated well with those from biochemical identification $^{13-17}$. Beyond the molecular techniques, the sequencing of the specific genes rpoB and hsp65 has become the gold standard for identifying mycobacteria ${ }^{18,19}$. Because of its high discriminatory power, this tool has been used by several groups in epidemiological studies to identify the species involved in human diseases and in outbreaks, and also for taxonomic reclassification ${ }^{20,21}$.

Multiplex polymerase chain reaction (PCR) is a fast tool that allows the simultaneous amplification of more than one sequence of target deoxyribonucleic acid (DNA) in a single reaction, saving time and reagents ${ }^{22-24}$. This system, which amplifies two or three different targets, can differentiate M. tuberculosis from the NTMs ${ }^{25,26}$. This molecular approach is used in identifying and differentiating microbes because it is able to supply a simple fingerprint of certain bacterial groups when compared to the standard profiles of referent strains. The correct choice of target sequences in the genome is one of the key criteria for detecting and identifying mycobacteria by $\mathrm{PCR}^{27}$.

Faced with the need to differentiate tuberculosis from other mycobacteria, the aim of this study was to evaluate a system based on multiplex PCR, already optimized by Poroca et al. ${ }^{26}$, for identifying mycobacterial species isolated from different clinical samples from patients diagnosed with pulmonary and extrapulmonary tuberculosis. This test, in addition to contributing to a rapid and accurate differential diagnosis between tuberculosis and other mycobacteria, will certainly assist in the early and appropriate therapeutic management of the patient.

\section{METHODS}

\section{Study population}

Thirty-seven male and female patients over the age of 12 years with pulmonary and extrapulmonary tuberculosis diagnoses, who were referred to public clinics in the metropolitan region of Recife of northeastern Brazil between March 2008 and December 2009, were chosen for this study. The types of biological samples collected were chosen based on the different clinical forms of the disease. The diagnosis of tuberculosis was made by the attending physician in accordance with the standards of the American Thoracic Society (ATS), in 2007, based on the isolation of Mycobacterium in culture from different samples and visualization of acid fast bacilli (AFB) in the smear microscopy ${ }^{28}$.

\section{Biological specimens}

Forty pulmonary and extrapulmonary samples were analyzed, including $26(65 \%)$ samples of pulmonary origin and $14(35 \%)$ samples of extrapulmonary origin, according to the following distribution: $23(57.5 \%)$ from sputum, 3 (7.5\%) from a bronchoalveolar lavage (BAL), 8 (20\%) from urine, 1 (2.5\%) from skin lesion aspiration, 3 (7.5\%) from pleural fluid (PL), $1(2.5 \%)$ from a node biopsy and $1(2.5 \%)$ from a bone biopsy. Clinical specimens were analyzed by bacilloscopy, culture, multiplex PCR and polymerase chain reaction restriction enzyme analysis (PRA-hsp65) to identify and differentiate mycobacterial species as described below.

\section{Smear microscopy}

The presence of acid fast bacilli in different clinical specimens was determined by Ziehl-Neelsen staining (Stewart, 1953) in the Public Health Laboratory of Recife (Unified Health System - SUS), in accordance with the guidelines of the Brazilian Ministry of Health ${ }^{29}$.

\section{Specimen processing}

In total, of 1-5 $\mathrm{ml}$ of various clinical specimens (except for the sterile samples collected) was processed using the modified Petroff method in $4 \% \mathrm{NaOH}^{29,30}$.

\section{Culture}

Cultures were performed in a Lowenstein-Jensen medium ${ }^{29,31}$ and mycobacterium species were identified using the following biochemical tests: selective inhibition by para-nitrobenzoic (PNB) acid and thiophene-2-carboxylic hydrazide (TCH) acid, niacin accumulation and heat-stable catalase at $68^{\circ} \mathrm{C}^{29}$. The cultures analyzed using the biochemical tests in this study were considered as references for comparison with the results obtained by multiplex PCR and PRA- $h s p 65$.

\section{DNA extraction}

DNA was extracted and purified from reference strains of Mycobacterium tuberculosis (H37Rv). In all, 40 species of mycobacteria were isolated on a Lowenstein-Jensen medium from clinical samples obtained from 37 patients diagnosed with pulmonary and extrapulmonary tuberculosis using the conventional method described by Sambrook et al. using a mixture of solvents and phenol chloroform ${ }^{32}$.

\section{Polymerase chain reaction and restriction enzyme analysis}

The polymerase chain reaction and restriction enzyme analysis (PRA-hsp65) were performed using the techniques described in Telenti et al. ${ }^{13}$ The identification was determined by comparing the sizes of the fragments with the algorithm described in the PRASITE site (http://app.chuv.ch/prasite/ index.html) ${ }^{17}$.

\section{Multiplex polymerase chain reaction}

In multiplex PCR, there are 3 pairs of primers, 1 pair for each target. The gene encoding the antigen of Mycobacterium tuberculosis $65 \mathrm{KDa}$, the dnaJ gene and the insertion element IS6110 were used in a single reaction with a mixture containing $10 \mathrm{mM}$ Tris- $\mathrm{HCl}$ (pH 8.3); $50 \mathrm{mM} \mathrm{KCl} ; 2.5 \mathrm{mM} \mathrm{MgCl} 2 ; 2 \mathrm{mM}$ dNTP; $2.5 \mathrm{U}$ of Taq DNA Polymerase (Invitrogen); and 20pmol, $50 \mathrm{pmol}$ and $10 \mathrm{pmol}$ of each oligonucleotide pair, respectively. A total of $2 \mu \mathrm{L}$ of DNA at a concentration of $20 \mathrm{ng} / \mu \mathrm{L}$ for each strain of mycobacteria was added to the reaction mixture for 
a final volume of $50 \mu \mathrm{L}$. These reaction parameters are in accordance with the conditions described by Poroca et al. ${ }^{26}$.

\section{Statistical analysis}

The value of Kappa was determined using SPSS statistical software for Windows (version 18; SPSS, Chicago, IL).

\section{Ethical considerations}

The research was approved by the Ethics Committee of the Aggeu Magalhães Research Center (CPqAM) and the respective ethics committees of the participating hospitals under protocol No. 39/2009.

\section{RESULTS}

\section{Clinical, epidemiological and laboratory findings}

Of the 37 patients diagnosed with tuberculosis, $48.7 \%$ $(n=18)$ of the cases originated from the ward, and $59.5 \%$ $(n=22)$ were males aged 12 to 64 years (median 39 years). The clinical results showed that $70.3 \%(n=26)$ of the patients had pulmonary tuberculosis. The main clinical symptoms found were weight loss (90\%), cough (80\%) and fever (62.5\%). Among the patients' pre-existing diseases, $24.3 \%(n=9)$ were infected with the human immunodeficiency virus (HIV) virus and $13.5 \%$ $(n=5)$ previously had tuberculosis. In laboratory tests, $82 \%$ had chest X-rays and 21.7\% had the Mantoux tuberculin skin test. A smear of lung samples was taken from $73 \%(n=19)$ of the patients; 17 were sputum samples and 2 were bronchoalveolar lavages, and $94.4 \%(n=18)$ of the specimens lung specimens were positive for The presence of acid fast bacilli in different clinical specimens was determined by Ziehl-Neelsen staining (Stewart, 1953) in the Public Health Laboratory of Recife (Unified Health System - SUS), in accordance with the guidelines of the Brazilian Ministry of Health. Mycobacterial strains were isolated in Lowenstein-Jensen culture medium for the 40 biological specimens analyzed (Table 1).

\section{Identification of the mycobacterial species by culture and biochemical testing (reference test)}

With respect to the reference test, of the 40 mycobacterial strains isolated by culture from the specimens obtained from the 37 patients diagnosed with tuberculosis, $57.5 \%(n=23)$ of the strains were identified as belonging to the M. tuberculosis complex, $20 \%(n=8)$ of the strains were identified as nontuberculous mycobacteria (NTM) and $22.5 \%(n=9)$ of the results were inconclusive.

\section{Evaluation of multiplex PCR using mycobacterial species}

Of the 40 mycobacterial isolates, 27 (67.5\%) showed the molecular pattern for $M$. tuberculosis with the simultaneous amplification of the three targets IS6110 (541bp), dnaJ (365bp) and $h s p 65$ (165bp). In 13 of the samples (32.5\%), amplification of a fragment sized 20 to 40 base pairs less than 165bp was observed, which characterized these samples as NTM, according to Poroca et al. ${ }^{26}$ (Figure 1).
TABLE 1 - Clinical, epidemiological and laboratory characteristics of the 37 patients studied.

\begin{tabular}{|c|c|c|c|c|}
\hline \multirow[b]{2}{*}{ Characteristic } & \multicolumn{2}{|c|}{ Pulmonary TB } & \multicolumn{2}{|c|}{ Extrapulmonary TB } \\
\hline & $\mathrm{n}$ & $\%$ & $\mathrm{n}$ & $\%$ \\
\hline Out-patients & 10 & 27.0 & 9 & 24.4 \\
\hline Ward & 16 & 43.2 & 2 & 5.5 \\
\hline \multicolumn{5}{|l|}{ Sex } \\
\hline male & 17 & 45.9 & 5 & 13.5 \\
\hline female & 9 & 24.3 & 6 & 16.2 \\
\hline \multicolumn{5}{|l|}{ Pre-existing disease } \\
\hline HIV & 1 & 2.7 & 5 & 13.5 \\
\hline TB re-treatment & 4 & 10.8 & 1 & 2.7 \\
\hline \multicolumn{5}{|l|}{ Laboratory exams } \\
\hline smear & 19 & 73.0 & 0 & 0.0 \\
\hline culture & 26 & 65.0 & 14 & 35.0 \\
\hline
\end{tabular}

HIV: human immunodeficiency virus; TB: tuberculosis.

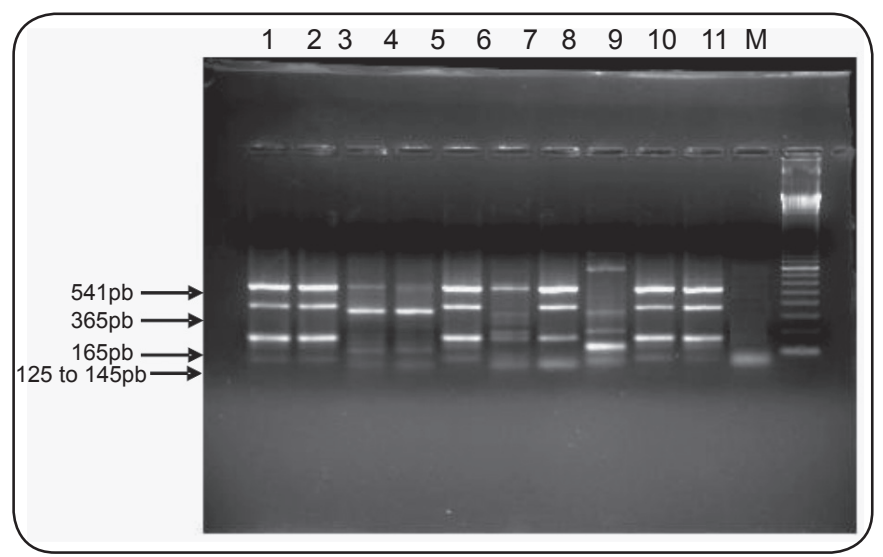

FIGURE 1 - Multiplex PCR of mycobacterial isolates from patients diagnosed with tuberculosis. Bands 1, 2, 5 and 7 represent the mycobacterial species displaying the molecular pattern of M. tuberculosis; Bands 3, 4, 6 and 8 represent the mycobacterial species displaying the molecular pattern of NTM; Bands 9 and 10 represent genomic DNA for M. tuberculosis (H37Rv); Band 11 represents a negative control for the reaction; Band $\mathrm{M}$ represents a 50bp DNA ladder (New England BioLabs, Hitchin, Hertfordshire, UK).

\section{Species identification using restriction enzyme analysis}

When the mycobacterial species were identified by analyzing the banding pattern obtained by the PRA-hsp65 technique, 29 (72.5\%) of the species were identified as the Mycobacterium tuberculosis complex; 4 (10\%) of the species as Mycobacterium fortuitum 2; one (2.5\%) of the species as Mycobacterium abscessus 2, Mycobacterium bolletii/Mycobacterium massiliense; one (2.5\%) of the species as Mycobacterium gastritis 2/M. kansasii 6 and one $(2.5 \%)$ of the species as Mycobacterium parmense type 1 , whereas $4(10 \%)$ of the results were inconclusive. None of the results indicated the presence of the $M$. avium complex or 
M. intracellulare among the strains isolated via culture from the pulmonary and extrapulmonary tuberculosis samples.

\section{Results of the multiplex PCR and PRA-hsp65 with strains whose results were inconclusive by the reference test (culture and biochemical testing)}

In this study, 9 (22.5\%) of the mycobacteria samples isolated in culture from the clinical specimens of patients diagnosed with tuberculosis could not be identified by culture and biochemical tests; however, $5(55.5 \%)$ of these strains were identified as M. tuberculosis by multiplex PCR, and 4 (44.4\%) were identified as NTM. When using the PRA-hsp65, $6(66.6 \%)$ strains were identified as an M. tuberculosis complex, and 3 (33.3\%) could not be identified by PRA-hsp 65 . The diagnosis of tuberculosis in these patients was performed by the medical health service based on smear and/or clinical symptoms.

\section{Comparison of the reference test with multiplex PCR and PRA-hsp65}

The results of the multiplex PCR and PRA-hsp65 were compared to the results of the reference test (culture and biochemical assays) and are summarized in Table 2. The correlation calculation between the two tests was conducted using the results from 30 strains identified by culture and biochemical tests, as these results were not considered inconclusive in the reference tests and PRA-hsp65.

TABLE 2 - Concordance between the results of multiplex PCR and PRA-hsp 65 with the biochemical and phenotypic tests considered for 30 different isolates of mycobacteria differentiated by phenotypic and biochemical tests.

\begin{tabular}{lcc}
\hline Biochemical and phenotypic tests & Multiplex PCR & PRA-hsp65 \\
\hline MTBC & 23 & 23 \\
NTM & 7 & 7 \\
Total & 30 & 30 \\
Kappa & 1 & 1 \\
\hline
\end{tabular}

MTBC: Mycobacterium tuberculosis complex; NTM: non-tuberculous mycobacteria. PCR: polymerase chain reaction; PRA-hsp 65: polymerase chain reaction and restriction enzyme analysis.

\section{Data from subjects who had mycobacteria identified as NTM by multiplex PCR and PRA-hsp65}

We found that $7(17.5 \%)$ strains of mycobacteria were identified as NTM using multiplex PCR and PRA- $h s p 65$ in 6 individuals who were diagnosed with pulmonary or extrapulmonary tuberculosis. Among those individuals, only one subject had two different isolates derived from clinical urine (non-sterile) and pleural fluid (sterile) specimens. The other 5 patients had only one species of NTM isolated in culture from the clinical urine, sputum or BAL specimens. None of these cases were diagnosed as an NTM infection.

\section{DISCUSSION}

Advances in molecular biology techniques and the information provided by sequencing the complete genome of M. tuberculosis prompted the development of new tools for the rapid diagnosis of tuberculosis, including the differentiation of M. tuberculosis from other mycobacteria ${ }^{33,34}$. Multiplex PCR has the ability to amplify different targets simultaneously and has been used to detect and identify mycobacteria from the Mycobacterium tuberculosis complex and nontuberculous mycobacteria ${ }^{26,27,35,36}$.

In this study, when the multiplex PCR was compared with the reference tests (cultures and phenotypic and biochemical tests) to identify 40 mycobacterial isolates, a significant agreement was observed: $22(100 \%)$ of the 37 cases with a final diagnoses of pulmonary or extrapulmonary tuberculosis presented species of the Mycobacterium tuberculosis complex (MTBC) using the reference tests. Multiplex PCR identified the presence of the M. tuberculosis complex in $27(67.5 \%)$ of the cases. In $13(32.5 \%)$ of the cases, multiplex PCR showed a molecular pattern consistent with an NTM, thus disagreeing with the final diagnosis made by the attending physician. It is important to be cautious when making a differential diagnosis between tuberculosis and other mycobacteria because the clinical symptoms and X-ray images may be similar. In Pernambuco in 2010 , approximately $26.7 \%$ of the patients were treated without bacteriological confirmation of pulmonary tuberculosis; the diagnosis was based only on clinical and X-ray findings, which are often inconclusive and provide inferior data relative to those obtained in this study ${ }^{37}$.

The results of the PRA-hsp65 technique, when compared with the reference tests, showed the same degree of agreement obtained with the multiplex PCR for the Mycobacterium tuberculosis complex and NTM. It is notable that the multiplex PCR, because of its ability to amplify three different, specific targets in the same reaction, has the ability to identify the Mycobacterium tuberculosis complex and differentiate it from the species M. tuberculosis, M. bovis and M. avium $^{26,27}$. In contrast, although PRA-hsp 65 does not differentiate between the species of the Mycobacterium tuberculosis complex, it is able to identify most of the NTM species ${ }^{5,38}$. This tool is recommended when the culture and phenotypic and biochemical tests suggest the presence of NTM species, but it is costly and time-intensive ${ }^{5}$.

During this study, 3 patients diagnosed with pulmonary tuberculosis were beginning their second dose of anti-TB treatment when the species of mycobacteria was identified as NTM by multiplex PCR. In 2 cases, only 1 isolation from a lung sample was obtained, and in one of the cases, the species was identified as M. gastritis 2/M. kansassi 6 using PRA-hsp65. The presence of NTM in a single sample from a non-sterile source requires careful investigation, including requests for new samples to eliminate the possibility of contamination or transitory colonization ${ }^{11,39}$. In this study, no patients were diagnosed by the health service professionals as infected with NTM; therefore, there is a need for patient follow-up using a 
new collection of biological specimens, as the diagnosis may be suspect. The present study showed that, despite the high prevalence of tuberculosis in Brazil, the presence of NTM should strongly be considered prior to growing mycobacterial cultures in sterile clinical samples as part of the investigation when patients have symptoms such as an undefined fever.

The findings in this study suggest the need to investigate the presence of NTM in diagnosed lung diseases, following from the recommendations of other studies, which showed that NTM species can be detected in cases where TB is being re-treated ${ }^{7,8}$. The most frequently isolated species belong to the $M$. avium (MAC) complex and M. kansassi ${ }^{40,41}$. Ueki et al. ${ }^{11}$ demonstrated that the most prevalent species in the lung compartment was M. kansassi and MAC in disseminated diseases ${ }^{11}$. For the treatment of pulmonary NTM infections, laboratory support is needed both to identify species and to determine the in vitro profile of resistance to antimicrobial agents. M. kansasii remains the most easily treatable form of pulmonary infections; there is a strong correlation between the in vitro susceptibility and the in vivo response to rifampicin, macrolides and fluoroquinolones. The greatest risk is the emergence of drug-resistant strains, similar to what is observed with M. tuberculosis ${ }^{42}$, when patients fail to follow the correct treatment regimen.

Among the cases diagnosed as extrapulmonary tuberculosis, we isolated the same species of mycobacteria (M. fortuitum 2) in Lowenstein Jensen (LJ) culture medium from samples of urine and pleural fluid, where the bacteriological diagnosis for NTM can be defined according to ATS criteria because the pleural fluid is considered a sterile sample ${ }^{28}$. The M. fortuitum 2 species were identified by phenotypic and biochemical tests, multiplex PCR and PRA-hsp65. M. fortuitum is a rapidly growing mycobacterium. Although its pathogenic potential is very low, it is often acquired in hospitals and can infect immunocompromised patients. One patient was HIV-positive and died as soon as he began treatment for tuberculosis, which indicates the increasing clinical importance of identifying mycobacterial species ${ }^{43,44}$. Several studies have demonstrate the importance of $M$. fortuitum infections in immunocompromised patients and found that the most prevalent species in these cases include the Mycobacterium avium complex, Mycobacterium kansasii and Mycobacterium fortuitum ${ }^{10,11,45}$.

It can therefore be concluded that multiplex PCR has the ability to identify and differentiate species of M. tuberculosis and NTMs and thus can be used as an auxiliary tool in the differential diagnosis of tuberculosis and diseases caused by NTMs. However, multiplex PCR should not be used as a sole laboratory method; it is important that the differential diagnosis be based on the joint analysis of various parameters using PRA-hsp65 tests and the sequencing of specific genes that may identify the NTM species.

The speed and accuracy of multiplex PCR to differentiate between the M. tuberculosis complex and NTMs enables it to be used as an alternative to phenotypic and biochemical tests when identifying species of the $M$. tuberculosis complex and when screening to determine the NTM species present by sequencing specific genes.
Thus, molecular methods (multiplex PCR, PRA- $h s p 65$ and sequencing of specific genes) can support diagnoses based on clinical determinations regarding mycobacterial infections, and the implementation of these methods, particularly in reference to services for those patients with tuberculosis (TB), Multidrug-resistant tuberculosis (MDR- TB) and Human Immunodeficiency Virus/ Acquired Immunodeficiency Syndrome (HIV/AIDS), reduces the possibility of an inadequate diagnosis and treatment.

\section{ACKNOWLEDGMENTS}

Karla Patrícia de Oliveira Luna reviewed the English version of this manuscript.

\section{CONFLICT OF INTEREST}

The authors declare that there is no conflict of interest.

\section{FINANCIAL SUPPORT}

This study received financial support from the Aggeu Magalhães Center for Research (Fiocruz-PE).

\section{REFERENCES}

1. American Thoracic Society. Diagnosis and treatment of disease caused by nontubeculous mycobacteria. Am J Respir Crit Care Med 1997; 156:1-25.

2. Tortoli E. Impact of genotypic studies on mycobacterial taxonomy: the new mycobacteria of the 1990's. Clin Microbiol Rev 2003; 2:319-354.

3. Yew WW, Sotgiu G, Migliori GB. Update in tuberculosis and nontuberculous mycobacterial disease 2010. Am J Respir Crit Care Med 2011; 184:180-185.

4. Barreto AM, Campos EC. "Non-tuberculous" mycobacteria in Brazil. Bull Pneumol. Sanit 2000; 8:23-32.

5. Chimara E. Avaliação de métodos moleculares para identificação de micobactérias e elaboração de um algoritmo de indentificação. [Thesis]. [São Paulo]: Universidade Federal de São Paulo; 2005. 134p.

6. Ministério da Saúde. Agência Nacional de Vigilância Sanitária. Relatório descrito de investigação de casos de infecção por micobactérias não tuberculosas de crescimento rápido (MCR) no Brasil no período de 1998 a 2009; Brasília: Ministério da Saúde; 2011.

7. Matos ED, Santana MA, Santana MC, Mamede P, Bezerra BL, Panão ED, et al. Nontuberculosis mycobacteria at a multiresistant tuberculosis reference center in Bahia: Clinical Epidemiological aspects. Braz J Infect Dis 2004; 8:296-304.

8. Costa ARF, Lopes ML, Leo SC, Schneider MPC, Sousa MS, Suffys PN, et al. Molecular identification of Rapidly growing mycobacteria isolates from pulmonary specimens of patients in the state of Pará, Amazon region, Brazil. Diagn Microbiol Infect Dis 2009; 65:358-364.

9. Valdés F, Cid A. Micobactérias atípicas. Actas Dermosifiliogr 2004; 95:331-357.

10. García- Martos P, García-Agudo L. Infecciones por micobacterias de crecimiento rápido. Enferm Infecc Microbiol Clin 2012; 30:192-200.

11. Ueki SYM, Martins MC, Telles MAS, Virgillio MC, Giampaglia CMS, Chimara E, et al. Nontuberculous mycobacteria: species diversity in São Paulo State, Brazil. J Bras de Patol Med Lab 2005; 41:1-8. 
12. Neonakis IK, Gitti Z, Krambovitis E, Spandidos DA. Molecular diagnostic tools in mycobacteriology. J Microbiol Methods 2008; 75:1-11.

13. Telenti A, Marchesi F, Balz M, Bally F, Bottger EC, Bodmer T. Rapid identification of mycobacteria to the species level by polymerase chain reaction and restriction enzyme analysis. J Clin Microbiol 1993; 31:175-178.

14. Devallois A, Goh KS, Rastogi N. Rapid identification of mycobacteria to species level by PCR-restriction fragment length polymorphism analysis of the hsp65 gene and proposition of an algorithm to differentiate 34 mycobacterial species. J Clin Microbiol 1997; 35:2969-2973.

15. Silva CF, Ueki SYM, Geiger DC, Leão SC. hsp65 PCR-Restricion enzyme analysis (PRA) for identification of mycobacteria in clinical laboratory. Rev Inst Med Trop 2001; 43:25-28.

16. Silva RA, Werneck BAM, Dias CCE, Villas-Boas SM, Fonseca L, Saad $\mathrm{MH}$, et al. Novel allelic variants of Mycobacteria isolated in Brazil as determined by PCR-restriction enzyme analysis of hsp65. J Clin Microbiol 2002; 40:4191-4196.

17. Chimara E. Realible identification of mycobacterial species by PCRrestriction enzyme análisis (PRA)- hsp65 in a referente laboratory and elaboration of a sequence-based extended algorithm of PRA-hsp65 patterns. BMC Biotechnol 2008; 20:8-48.

18. Cheml ALKC, Portaels F. Molecular diagnosis of nontuberculous myobacteria. Curr Opin Infect Dis 2003; 16:77-83.

19. Herrera-León L, Pozuelo-Díaz R, Moreno TM, Cobacho AV, Pilar SV, Pajares MSJ. Aplicación de métodos moleculares para la identificación de las espécies del complejo Mycobacterium tuberculosis. Enferm Infecc Microbiol Clin 2009; 27:496-502.

20. Leão SC, Tortoli E, Viana-Niero C, Ueki SYM, Lima KVB, Lopes ML, et al. Characterization of Mycobacteria from a Major Brazilian Outbreak Suggests that Revision of the Taxonomic Status of Members of the Mycobacterium chelonae-M. abscessus Group Is Needed. J Clin Microbiol 2009; 2691-2698.

21. Duarte RS, Lourenço MC, Fonseca LS, Leão SC, Amorim EL, Rocha IL, et al. Epidemic of postsurgical infections caused by Mycobacterium massiliense. J Clin Microbiol 2009; 47:2149-2155.

22. Hernandez M, Rodríguez-Lázaro D, Esteve T, Prat S, Pla M. Development of melting temperature-based SYBR Green I polymerase chain reaction methods for multiplex genetically modified organism detection. Anal Biochem 2003; 323:164-170.

23. Tanaka II, Anno IS, Leite SRA, Cooksey RC, Leite CQF. Comparison of a multiplex-PCR assay with mycolic acids analysis and conventional methods for the identification of mycobacteria. Microbiol Immunol 2003; 47:307-312.

24. Corsetti A, Settanni L, Valmorri S, Mastrangelo M, Suzzi G. Identification of subdominant sourdough lactic acid bacteria and their evolution during laboratory-scale fermentations. Food Microbiol 2007; 24:592-600.

25. Kim H, Mun H, Kim H, Oh E, Ha Y, Bai G, et al. Differentiantion of mycobacterial species by hsp65 duplex PCR followed by duplex-PCRbased restriction analysis and direct sequencing. J Clin Microbiol 2006; 44:3855-3862.

26. Poroca DR, Lima AS, Lima JFA, Cruz HLA, Montenegro RA, Melo FL, et al. Diferenciação de micobactérias por PCR multiplex. Rev Soc Bras Med Trop 2009; 42:716-722.

27. Bhattacharya A, Karak K, Ghosal AG, Roy A, Das S, Dandapat P, et al. Development of a new sensitive and efficient multiplexpolymerase chain reaction $(\mathrm{PCR})$ for identification and differentiation of different mycobacterial species. Trop Med Int Health 2003; 8:150-157.

28. American Thoracic Society Documents. An Official ATS/IDSA statement: diagnosis, treatment and prevention of nontuberculous mycobacterial diseases. Am J Respir Crit Care Med 2007;175:367-416.

29. Ministério da Saúde. Secretaria de Vigilância em Saúde em Saúde. Manual do Laboratório Nacional de Vigilância da Tuberculose e outras micobactérias, Brasília: Ministério da Saúde; 2008.

30. Kent PT, Kubica GP. Public Health Mycobacteriology: A Guide for the Level III Laboratory. Department of Health and Human Services, Atlanta, GA, U.S: Centers for Disease Control; 1985.

31. Liu PI, McGregor DH, Faucher I, Jinks WL, Miller LA, Green L, et al. Comparison of three culture media for isolation of Mycobacterium tuberculosis: a 6-year study. Appl Microbiol 1973; 26:880-883.

32. Sambrook J, Fritsch EF, Maniatis T. Molecular Cloning. A Laboratory Manual. 2nd ed. Cold Spring Harbor: Cold Spring Harbor Laboratory Press; 1989.

33. Cole ST, Brosch R, Parkhill J, Garnier T, Churcher C, Harris D, et al. Deciphering the Biology of Mycobacterium tuberculosis from the Complete Genome Sequence, Nature 1998; 393:537-544.

34. Palomino JC. Nonconventional and new methods in the diagnosis of tuberculosis: feasibility and applicability in the field. Eur Respir J 2005; 26:339-350.

35. Klemen HMD, Bogiatzis AMD, Ghalibafian MMD, Popper HH. Multiplex polymerase chain reaction detection of atypical mycobacteria and Mycobacterium tuberculosis complex. Diagn Mol Pathol 1998; 7:310:316.

36. KIM BJ, Hong SK, Lee KH, Yun YJ, Kim EC, Park YG, et al. Differential identification of Mycobacterium tuberculosis complex and nontuberculous mycobacteria by duplex PCR assay using the RNA polymerase gene (rpoB). J Clin Microbiol 2004; 42:1308-1312.

37. Ministério da Saúde. Boletim Epidemiológico Secretaria de Vigilância em Saúde. Volume 43. Brasília: Ministério da Saúde; 2012.

38. Brunello F, Ligozzi M, Cristelli E, Bonora S, Tortoli E, Fontana R. Identification of 54 mycobacterial species by PCR-restriction fragment length polymorphism analysis of the $h s p 65$ gene. J Clin Microbiol 2001; 39:2799-806.

39. Van Crevel R, Lange WC, Vanderpuye NA, Van Soolingen D, HoogkampKorstanje JA, Van Deuren KM, et al. The impact of nontuberculous mycobacteria on management of presumed pulmonary tuberculosis. Infection 2001; 29(2):59-63.

40. García JMG, Gutiérrez PJJ, Sánchez AAA. Respiratory infections caused by environmental mycobacteria. Arch Bronconeumol 2005; 41:206-219.

41. Gomez NA. Non-tuberculous mycobacteria: an emerging infection? An Pediatr 2009; 71:185-188.

42. Griffith DE, Aksamit TR. Therapy of refractory nontuberculous mycobacterial lung disease. Curr Opin Infect Dis 2012; 25:218-227.

43. Hinrichsen SL. Micobacteria de crescimento rápido - MRC. Prática Hospitalar 2007; 53:106-111.

44. Phillips MS, Von Reyn CF. Nosocomial infections due to non-tuberculous mycobacteria. Clin Infect Dis 2001; 33:1363-1374.

45. Ngueyn DQ, Righini C, Darauassi Y, Schmerber S. Nasal infection due to Mycobacterium fortuitum. Eur Ann Otorhinolaryngol Head Neck Dis 2011; 128:197-199. 